\title{
A research institute at a turning point
}

T he San Raffaele del Monte Tabor Foundation in Milan, Italy, manages a multidisciplinary hospital and research institute, renowned as a center of excellence for biomedical research. Early in 2011, it was announced that the institute was in a precarious financial position, on the brink of bankruptcy. In October, a Milan government tribunal approved a restructuring plan that involves a financial takeover of the institute by the Vatican Bank and a private investor. The JCI recently spoke to Maria Grazia Roncarolo, the scientific director at San Raffaele, about this crisis and the institute's future.

JCI: What makes the San Raffaele Scientific Institute different from other European research centers?

Roncarolo: We have a very large hospital and a research institute, which belong to the San Raffaele Foundation, and a private university, the San Raffaele Vita-Salute University, which is a separate legal entity. Research activities at the hospital, the research institute, and the university are well integrated. Most of our research programs move from the bench to the bedside and back. Our "brand" is translational research. These days translational research is very fashionable, but we've been doing it for many years. We currently have approximately 570 clinical trials ongoing, with about 10,500 patients involved. Of these trials, more than half are based on our own research.

JCI: As the scientific director, what is your vision for the future of the institute?

Roncarolo: We want to maintain our excellence in neuroscience, genetic diseases, and immunology-based diseases, and we want to expand our research in oncology and cardiovascular diseases, two disease areas for which the hospital projects its biggest growth. Further advancing our cutting-edge translational research will be another key priority. This is absolutely necessary to continue to deliver world-class personalized medicine in the future. We also want to keep a very strong focus on the training of physician-scientists, to provide them with a scientific infrastructure, which will allow them to develop and flourish.

JCI: How did you find out about the financial crisis at the institute?

Roncarolo: As long as I've been here, I've heard that we were in debt, but the scientific community of the institute was not aware

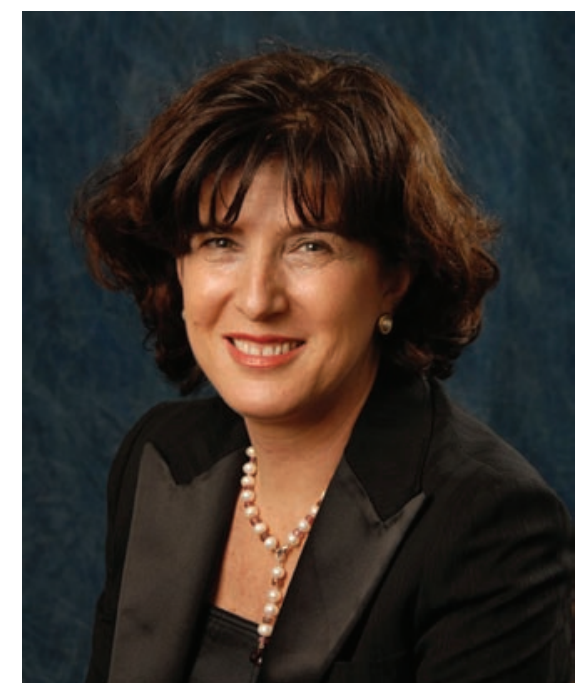

ficult. Now that the tribunal has approved a restructuring plan, I'm hopeful that the worst is behind us and that we'll be able to return to a certain degree of normalcy.

JCI: What do you think about the possibility of a Vatican Bank bailout, and are you concerned about that organization having influence over the direction of the research?

Roncarolo: I think that one of the biggest strengths of the institute is that we operate with the greatest degree of freedom in our research and clinical activities. That doesn't mean a lack of governance. It means that we value our intellectual freedom. We strongly hope that this will be maintained with the present and future owners. Presently, the board consists of four people appointed by the Vatican. We are happy that they are here. They put on the table a concrete offer that allowed the tribunal to approve a restructuring plan. We really hope that the new board will keep the spirit of this place, which is scientific freedom and a high level of autonomy for innovative basic and clinical research. We hope that the merit-driven culture and the conviction that research provides the backbone of the institute will continue. We also hope that the new for-profit structure planned for San Raffaele's core activities will not result in a separation of the basic and clinical research and teaching activities that, as a result of our hard work in the last years, are now fully and efficiently integrated. Such separation would make us lose our competitive edge in translational medicine. It is very clear that state-of-the-art clinical treatments are science driven and, if we would like to stay at the forefront of global developments in personalized medicine, basic and clinical research have to work side by side.

The San Raffaele Scientific Institute is internationally a highly regarded center of excellence, based on its scientific productivity, the number of innovative clinical trials, the substantial external funding obtained through competitive grants, and the international standing of its scientists. This excellence is still there, and the financial crisis didn't destroy it. We really hope that the new owners will realize the value of the institute and that they have the wisdom to enable us to further strengthen our international competitiveness and to support our scientific freedom.

\section{Kathryn Claiborn}

